\title{
Topiramate Protects Pericytes from Glucotoxicity: Role for Mitochondrial CA VA in Cerebromicrovascular Disease in Diabetes
}

\author{
Ping Patrick ${ }^{1}$, Tulin O. Price ${ }^{1}$, Ana L. Diogo ${ }^{1}$, Nader Sheibani ${ }^{2}$, William A. Banks ${ }^{3}$ and Gul N. Shah ${ }^{1 *}$ \\ ${ }^{1}$ Division of Endocrinology, Department of Internal Medicine, Saint Louis University School of Medicine, Edward A. Doisy Research \\ Center, 1100 South Grand Blvd, DRC 354, Saint Louis, MO 63104, USA \\ ${ }^{2}$ Ophthalmology and Visual Sciences, University of Wisconsin School of Medicine and Public Health, Madison, Wisconsin, USA \\ ${ }^{3}$ Division of Gerontology and Geriatric Medicine, Department of Medicine, VAPSHCS/GRECC S-182, Building 1, Room 810A, 1660 S. \\ Columbian Way, Seattle, WA 98108, University of Washington, Seattle, WA, USA
}

Received: March 31, 2015; Accepted: May 04, 2015; Published: May 20, 2015

*Corresponding author: Gul N. Shah, Division of Endocrinology, Department of Internal Medicine, Saint Louis University School of Medicine, Edward A. Doisy Research Center 1100 South Grand Blvd, DRC 315, St. Louis, MO 63104, Tel: +1 314977 9293; Fax: +1 314977 6797; Email: shahgn@slu.edu

\begin{abstract}
Hyperglycemia in diabetes mellitus causes oxidative stress and pericyte depletion from the microvasculature of the brain thus leading to the Blood-Brain Barrier (BBB) disruption. The compromised BBB exposes the brain to circulating substances, resulting in neurotoxicity and neuronal cell death.
\end{abstract}

The decline in pericyte numbers in diabetic mouse brain and pericyte apoptosis in high glucose cultures are caused by excess superoxide produced during enhanced respiration (mitochondrial oxidative metabolism of glucose). Superoxide is precursor to all Reactive Oxygen Species (ROS) which, in turn, cause oxidative stress. The rate of respiration and thus the ROS production is regulated by mitochondrial carbonic anhydrases (mCA) VA and VB, the two isoforms expressed in the mitochondria.

Inhibition of both mCA: decreases the oxidative stress and restores the pericyte numbers in diabetic brain; and reduces high glucose-induced respiration, ROS, oxidative stress, and apoptosis in cultured brain pericytes. However, the individual role of the two isoforms has not been established. To investigate the contribution of mCA VA in ROS production and apoptosis, a mCA VA overexpressing brain pericyte cell line was engineered. These cells were exposed to high glucose and analyzed for the changes in ROS and apoptosis. Overexpression of mCA VA significantly increased pericyte ROS and apoptosis. Inhibition of mCA VA with topiramate prevented increases both in glucose-induced ROS and pericyte death.

These results demonstrate, for the first time, that mCA VA regulates the rate of pericyte respiration. These findings identify mCA VA as a novel and specific therapeutic target to protect the cerebromicrovascular bed in diabetes.

Keywords: Diabetes mellitus; Brain pericytes; Apoptosis; Mitochondrial CA VA; Topiramate

\section{Abbreviations}

BBB: Blood-brain barrier; BPC: Brain Pericytes; mCA: Mitochondrial carbonic anhydrases; CAVA-BPC: Mitochondrial CA VA Over Expressing Brain Pericyte Cell Line; ETC: Electron Transport Chain; $\mathrm{HCO}_{3}$ : Bicarbonate; HG: High Glucose; NG: Normal Glucose; qRT-PCR: Quantitative Real Time PCR; ROS: Reactive Oxygen Species; TOP: Topiramate; TUNEL: Terminal Deoxynucleotidyl Transferase-dUTP Nick End Labeling

\section{Introduction}

Diabetes mellitus in addition to being a risk factor for Alzheimer's disease and stroke has its own pattern of cognition impairment where executive function in particular is affected [1-3]. Diabetes associated hyperglycemia attacks the microvasculature of insulin-insensitive tissues such as the eye, nerve, and brain [4]. Well known for causing blindness [5] and neuropathies [6], the microvascular disease induced by diabetes is also responsible for disruption of the Blood-Brain Barrier (BBB). The disruption leads to the loss of the BBB's ability to protect the brain from circulating substances, resulting in neurotoxicity and neuronal cell death [7].

The BBB is physically made up of endothelial cells of the microvasculature. However, pericytes in close proximity to endothelial cells interact with the latter and are vital for inducing and maintaining the integrity and function of the BBB [8]. Pericyte loss has been shown to cause disruption of the BBB in Alzheimer's disease [9]. Hyperglycemia induces pericyte loss in the mouse brain [10] and brain pericytes grown in high glucose undergo apoptosis [11]. Pericyte demise both in vivo and in vitro is triggered by access superoxide, produced during accelerated respiration (mitochondrial oxidative metabolism of glucose) $[12,13]$. Superoxide is precursor to all Reactive Oxygen Species 
(ROS) [14], which cause oxidative stress leading to pericyte death $[15,16]$. Brain pericyte cultured in high glucose exhibit significant increases in respiration, ROS, oxidative stress, and apoptosis $[11,13]$.

The rate of respiration and thus ROS and consequent pericyte apoptosis is regulated by mitochondrial carbonic anhydrases (mCA) VA and VB [17]. Of the 12 known active carbonic anhydrases only two that is VA and VB are expressed in the mitochondria. These isoforms provide bicarbonate $\left(\mathrm{HCO}_{3}^{-}\right)$for conversion of pyruvate to oxaloacetate, a key intermediate in respiration [13].

Genetic silencing of both mCA reduces oxidative stress in the mouse brain. In vivo, pharmacological inhibition of both mCA restores pericyte numbers depleted by hyperglycemia. In culture, pharmacological inhibition of both mCA slows the rate of respiration, ROS production, and pericyte apoptosis [13].

The individual contributions of mCA VA and mCA VB in pericyte ROS and apoptosis have not been investigated. Therefore, this study was designed to determine whether mCA VA plays a predominant role in the regulation of respiration. To accomplish this goal, a mCA VA overexpressing mouse brain pericyte cell line (CA VA-BPC) was established by stably transfecting the brain pericytes (BPC) with mCA VA cDNA. These cells were challenged with high glucose and mCA VA was inhibited pharmacologically with topiramate. The effects of mCA VA inhibition on intracellular ROS and pericyte apoptosis were determined.

We now report, for the first time, that the overexpression of mCA VA significantly increased intracellular ROS and apoptosis of pericytes. As expected both ROS and the percent of apoptotic pericytes were significantly reduced upon inhibition of mCA VA. These data demonstrate that mCA VA is an important pathway in the regulation of high glucose-induced ROS production and pericyte death and provides a novel and unique therapeutic target to protect the brain from hyperglycemia induced damage.

\section{Materials and Methods}

\section{Cell culture}

Conditionally immortalized mouse brain pericyte (BPC) cultures were established as previously described [11]. The pericytes were grown in $60 \mathrm{~mm}$ petri dishes in growth media (DMEM, D6046, Sigma-Aldrich, Saint Louis, MO) supplemented with $10 \%$ fetal bovine serum, $2 \mathrm{mM}$ L-glutamine, penicillin/ streptomycin (Sigma-Aldrich) and murine recombinant interferon- $\gamma$ at $44 \mathrm{U} / \mathrm{ml}$ (R\&D Systems, Minneapolis, MN) in an atmosphere of $5 \% \mathrm{CO}_{2}$ at $33^{\circ} \mathrm{C}$. The cells were fed every 2-3 days.

\section{Expression of mitochondrial CA VA in the brain pericytes}

Plasmid preparation: To show the effect of overexpression of mCA VA on pericyte ROS production and apoptosis, we developed mCA VA overexpressing cell line as follows: A 900 base pair coding sequence of mCA VA cDNA was directionally cloned into pDream2.1 (GenScript, Piscataway, NJ) mammalian expression vector (GenScript, Piscataway, NJ) at Bam HI/Hind III sites (Figure 1). pDream vector was selected because it lacks an SV40 origin. Immortalized pericytes express simian virus (SV40) large $\mathrm{T}$ antigen (tsA58Tag). A plasmid with an SV40 origin is not suitable for stable transfection of such cells. DNA rearrangements such as deletions and duplications found within and near integrated SV40 DNA in cell overexpressing large T antigen change the cells morphologically and physiologically [18].

Transfection: The BPC were transfected with Lipofectamine transfection reagent (Life technologies, Carlsbad, CA) according to supplier's protocol. Fifty five hours post transfection, cells were incubated in growth media containing $0.8 \mathrm{mg} / \mathrm{ml}$ Geneticin (Life technologies, Carlsbad, CA), to select for mCA VA overexpressing cells. Media was changed every 2-3 days. Expression of mCA VA mRNA was determined by quantitative real time PCR (qRT-PCR) and the level of mCA VA protein was assessed by immunoblot. Once established, mCA VA overexpressing cells (CA VA-BPC), were maintained in Geneticin containing media. Figure 1A shows that overexpression of mCA VA did not alter the morphology of the pericytes.

RNA isolation and quantitative real-time PCR: Total RNA was isolated from cultured pericytes using RNeasy kit (QIAGEN, Valencia, CA). For mRNA quantification, complementary DNAs (cDNAs) were synthesized using Superscript III first Strand Synthesis System (Invitrogen, Carlsbad, CA), following the manufacturer's protocol. Real time qPCR was performed in triplicate dishes of cells using Power SybrGreen reagent (Applied Biosystems, Carlsbad, CA) in a LightCycler-480 (Roche). The levels of mRNA were normalized to 36B4 as a housekeeping gene and calculated using the comparative $\Delta \mathrm{Ct}$ method. The sequences of the primer used were: mCA VA forward primer 5' - CCA GTC CAG AGG GGG TGT-3' and reverse primer 5'-CTG GCG TTT CCA GCA TTC-3'. There was a 50-fold increase in mCA VA mRNA in CA VA-BPC compared to parent BPC.

\section{Immunoblot analysis}

Immunoblotting was performed by standard procedures as previously described [10]. Briefly, cultured BPC and CA VA-BPC were homogenized in lysis buffer [25 mM Tris ( $\mathrm{pH} 7.5$ ), 0.15 $\mathrm{M} \mathrm{NaCl}, 1 \mathrm{mM}$ PMSF], sonicated, and cleared by centrifugation. Protein concentration in the final supernatants was determined by BCA Protein Assay (Pierce, Rockford, IL). The proteins (25 $\mu \mathrm{g}$ ) were separated on 4-12\% Bis-Tris reducing gels (NuPAGE Novex)
A

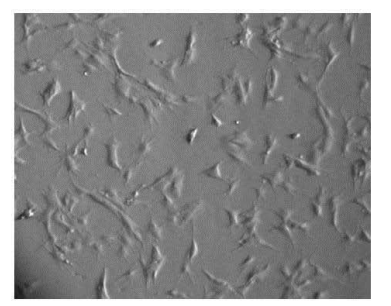

B

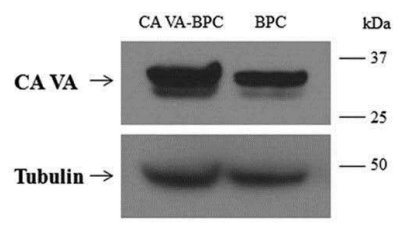

Figure 1: Immunoblot analysis of mCA VA protein in primary and CA VA over expressing pericytes. A) Light microscopic image of CA VA-BPC B) mCA VA polypeptide in BPC and CA VA-BPC. 
and transferred to nitrocellulose membranes. Polypeptides were identified by probing with mCA VA [19] and gamma-tubulin (MA1-850, Cell Signaling Technology, Danvers, MA) primary antibodies and horseradish peroxidase-conjugated secondary antibodies and visualized by chemiluminescent substrate (Pierce, Rockford, IL). Overexpressing CA VA-BPC showed 50\% more mCA VA protein compared to parent BPC (Figure 1B).

\section{Reactive Oxygen Species (ROS) Analysis}

Intracellular ROS were measured with a ROS activity assay kit (Cell Meter ${ }^{\mathrm{TM}}$ Fluorimetric Intracellular Total ROS activity assay kit, cat\# 22900, AAT Bioquest, Thermo Fisher Scientific, Inc., Waltham, MA) as described previously (13). Briefly, the BPC or CA VA-BPC were seeded in Costar black wall/clear bottom 96well plate at a density of $1 \times 10^{5}$ cells per well in $100 \mu \mathrm{l}$ of growth media containing normal glucose $(5.7 \mathrm{mM})$, and were allowed to adhere overnight in a $5 \% \mathrm{CO}_{2}, 37^{\circ} \mathrm{C}$ incubator. The following morning, $10 \mu \mathrm{M}$ of topiramate (TOP, T0575, Sigma-Aldrich) was added and incubations continued for 3 more hours. At the end of the treatment, $100 \mu \mathrm{l}$ of glucose stock solution (1 M) was added to bring the final concentration of glucose to $40.7 \mathrm{mM}$ (high glucose). Immediately after that, $100 \mu$ l of assay loading solution was added to each well and the incubations were continued in a $5 \% \mathrm{CO}_{2}, 37^{\circ} \mathrm{C}$ incubator for 1 hour. Fluorescence at excitation and emission wavelengths of 490 and $520 \mathrm{~nm}$, respectively, were measured using a fluorescence plate reader (Tecan Safire II, Tecan, Männedorf, Switzerland). Hydrogen peroxide was used as a positive control and Tempo as a negative control per the supplier's instructions. The ROS produced are presented as a percent of control. Each sample was run in triplicate and experiments were repeated at least three times.

\section{Cell viability}

A Cell Meter ${ }^{\mathrm{TM}}$ Cell Viability Assay Kit (cat\#22784, AAT Bioquest, Thermo Fisher Scientific, Inc.) was used to determine cell viability as described previously (13). Briefly, the BPC or CA VA-BPC were seeded in the wells of a Costar black wall/ clear bottom 96-well plate and treated with normal glucose, high glucose, and high glucose with and without topiramate, as described earlier. Following the treatment, the CytoCalcein Violet 450, AM dye-loading solution $(100 \mu \mathrm{l})$ was added and the cells were incubated in a $5 \% \mathrm{CO}_{2}, 37^{\circ} \mathrm{C}$ incubator for 1 hour. Fluorescence intensity was measured on a fluorescence plate reader (Tecan Safire II) at excitation and emission wavelengths of $405 \mathrm{~nm}$ and $460 \mathrm{~nm}$, respectively. The data is expressed as percentage of cell viability compared to viable cells in normal glucose. Each sample was run in triplicate and experiments were repeated at least three times.

\section{Programmed cell death analysis}

Apoptotic cell death was determined by TUNEL (Terminal deoxynucleotidyl transferase-dUTP nick end labeling) assay. The pericytes were grown for 5 days on poly L-lysine coated cover slips (neuVitro, El Monte, CA) under normal glucose, high glucose or high glucose containing $10 \mu \mathrm{M}$ of TOP. Following incubation, cells were fixed with $4 \%$ paraformaldehyde for $15 \mathrm{~min}$ at room temperature and washed with PBS twice. TUNEL staining was performed using Click-iT® TUNEL Alexa Fluor ${ }^{\circledR}$ 594, as recommended by the supplier (Invitrogen, Carlsbad, CA). Cells were counterstained with DAPI (Electron Microscopy Sciences, Hartfield, PA) for the nuclei and photographed using a Zeiss fluorescence microscope (Axiophot, Zeiss, Germany) equipped with a digital camera. TUNEL-positive cells were counted and calculated as a percentage of the total cells. The results are from three independent experiments.

\section{Statistical analysis}

All means are reported with their $n$ and SEM. Two means were compared by the unpaired two-tailed Student's $t$ test. For more than two means, ANOVA, followed by followed by a multiple group comparison test (Newman-Keuls) was used. $P<0.05$ was considered significant. Statistical analyses were made using GraphPad Prism 5.0 package program (GraphPad Software Inc, San Diego, CA).

\section{Results}

\section{Effect of mitochondrial CA VA overexpression on high glucose-induced intracellular pericyte reactive oxygen species (ROS)}

As shown in Figure 2 and Table 1, high glucose induced ROS in both BPC (NG, $100.0 \pm 4.6$ vs HG, $135.4 \pm 5.7, p<0.05)$ and $\mathrm{CA}$ VA-BPC (NG, $111.0 \pm 10.8$ vs HG, $165.2 \pm 16.8, p<0.01)$. However, the amount of ROS produced in CA VA-BPC was significantly higher than the parent BPC (HG, $165.2 \pm 16.8$ vs HG, $135.4 \pm 5.7$, respectively, $p<0.05$ ). Topiramate treatment blocked the effect of high glucose on ROS production in both BPC (HG, $135.4 \pm 5.7$ vs $\mathrm{HG}+10 \mu \mathrm{M}$ TOP, $80.0 \pm 9.5, \mathrm{p}<0.01)$ and CA VA-BPC (HG, $165.2 \pm$ 16.8 vs $\mathrm{HG}+10 \mu \mathrm{M}$ TOP, $75.2 \pm 8.1, p<0.001$ ) (Figure 2 and Table $1)$.

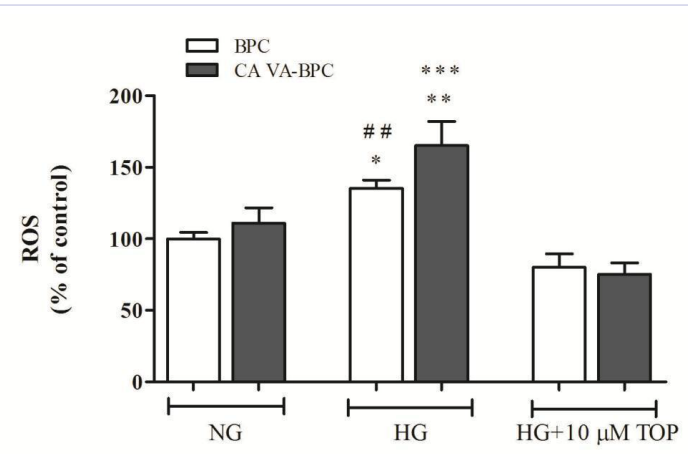

Figure 2: Effect of mCA VA overexpression on high glucose-induced intracellular Reactive Oxygen Species (ROS) in pericytes. Results are presented as percentage of BPC treated with NG. Data are shown as mean \pm SEM ( $n=6-7)$. The graphs are representative of three independent experiments. ${ }^{*} p<0.05$ for difference from NG-BPC and HG-CA VA-BPC, ${ }^{* *} p<0.01$ for difference from NG-CA VA-BPC, $\# \# p<0.01$ for difference from $\mathrm{HG}+10 \mu \mathrm{M}$ TOP-BPC, ${ }^{* * *} p<0.001$ for difference from $\mathrm{HG}+10 \mu \mathrm{M}$ TOP-CA VA-BPC. HG: High Glucose; NG: Normal Glucose; TOP: Topiramate. 
Table 1: Effect of Mitochondrial CA VA Overexpression on High GlucoseInduced Intracellular ROS in pericytes.

\begin{tabular}{|c|c|c|}
\hline Groups & BPC & CA VA-BPC \\
\hline NG & $100.0 \pm 4.6$ & $111.0 \pm 10.8$ \\
\hline HG & $135.4 \pm 5.7^{*, \# \#}$ & $165.2 \pm 16.8^{* *}, * * *$ \\
\hline HG+ 10 $\mu$ M TOP & $80.0 \pm 9.5$ & $75.2 \pm 8.1$ \\
\hline
\end{tabular}

Results are presented as percentage of BPC treated with NG. Data are shown as mean \pm SEM, $(n=6-7) .{ }^{*} p<0.05$ for difference from NG-BPC and HG-CA VA-BPC, ${ }^{* *} p<0.01$ for difference from NG-CA VA-BPC, \# \#p<0.01 for difference from $\mathrm{HG}+10 \mu \mathrm{M}$ TOP-BPC, ${ }^{* * *} p<0.001$ for difference from $\mathrm{HG}+10 \mu \mathrm{M}$ TOP-CA VA-BPC group. NG: normal glucose; HG: high glucose, TOP: Topiramate.

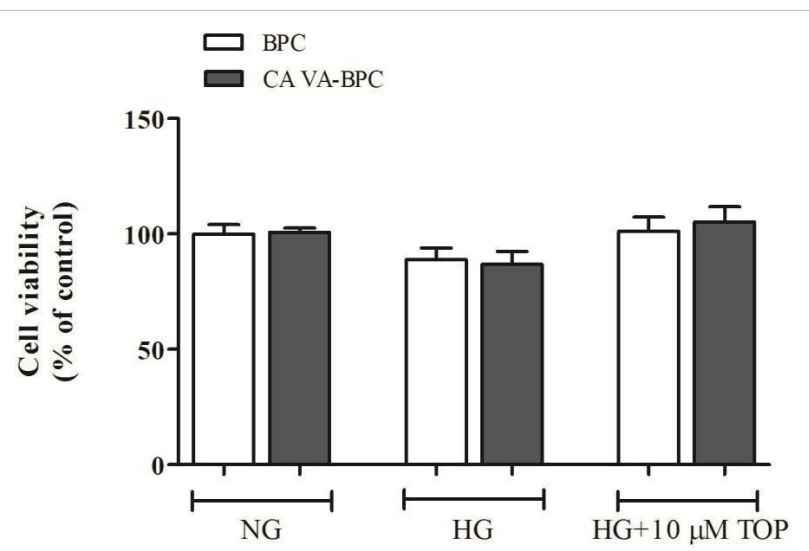

Figure 3: Cell viability. Cell viability is shown as percentage of viable cells in NG. Data are shown as mean \pm SEM $(n=6-7)$. The graphs are representative of three independent experiments. HG: High Glucose; NG: Normal Glucose; TOP: Topiramate.

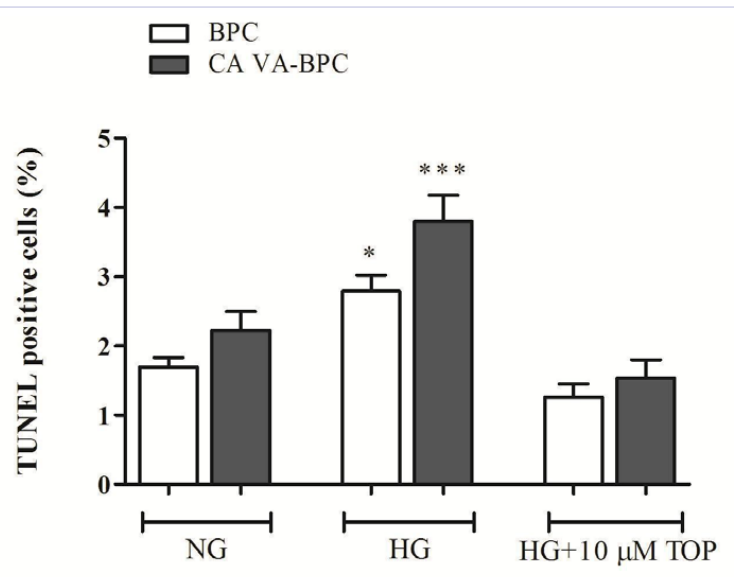

Figure 4: Effect of mCA VA overexpression on programmed cell death. A significant increase in TUNEL-positive cells induced by HG, was reversed upon treatment with TOP. The TUNEL positive cells are presented as a percentage of total cells. The values are expressed as mean \pm SEM $(\mathrm{n}=3) .{ }^{*} p<0.05$ for difference from NG-BPC, $\mathrm{HG}+10 \mu \mathrm{M}$ TOP-BPC, and HG-CA VA-BPC, ${ }^{* * *} p<0.001$ for difference from NG-CA VA-BPC and HG+10 $\mu$ M TOP-CA VA-BPC. HG: High Glucose; NG: Normal Glucose; TOP: Topiramate.

\section{Cell viability}

Both BPC and CA VA-BPC remained viable throughout the duration of the ROS determination assay in all treatment groups (Figure 3).

\section{Effect of mitochondrial CA VA overexpression on high glucose-induced pericyte apoptosis}

As expected, there was a significant increase in apoptosis in BPC exposed to high glucose. However, the number of apoptotic cells in CA VA-BPC in high glucose was even significantly higher than the parent BPC (Figure 4). Topiramate treatment rescued both BPC and CA VA-BPC from glucotoxicity (Figure 4).

\section{Discussion}

The significance of mCA VA and VB in diabetic disease of the central nervous system was recognized upon realization that: mCA double knockout mice exhibit reduced oxidative stress in the brain [10]; and pharmacological inhibition of these enzymes reduces hyperglycemia-induced oxidative stress and pericyte loss in diabetic mouse brain [10]. Pericyte in the microvasculature of the brain are vital to the integrity and function of endothelial cells which physically constitute BBB. Pericyte loss results in BBB disruption and cognitive decline [9]. Further investigations revealed that $\mathrm{mCA}$ regulate the rate of respiration, ROS production, and consequent oxidative stress leading to pericyte apoptosis $[10,11]$.

Oxidative stress is caused by overproduction of superoxide during excess respiration triggered by unrestricted influx of glucose in insulin-insensitive tissues such as the brain, eye, kidney, and nerve [4]. Mitochondrial CA regulate the rate of respiration as described in our earlier publications $[11,13]$ and in Figure 5. Briefly, glucose in the cytosol is metabolized to pyruvate by glycolysis. Pyruvate enters the mitochondria where it is carboxylated to oxaloacetate, a key enzyme in the Krebs cycle and Electron Transport Chain (ETC) reactions.

The $\mathrm{HCO}_{3}^{-}$required for carboxylation of pyruvate has to be produced inside the mitochondria; it cannot be imported from the cytosol because mitochondrial membranes are impermeant to $\mathrm{HCO}_{3}$. Mitochondrial CA, the carbonic anhydrases inside the mitochondria, provide $\mathrm{HCO}_{3}^{-}$by reversible hydration of carbon dioxide $\left(\mathrm{CO}_{2}+\mathrm{H}_{2} \mathrm{O} \Leftrightarrow \mathrm{HCO}_{3}^{-}+\mathrm{H}^{+}\right)$. Oxaloacetate, upon entering the Krebs cycle, generates electron donors, $\mathrm{FADH}_{2}$ and $\mathrm{NADH}$, which are carried to ETC to generate ATP. Superoxide is produced as a byproduct of ETC reactions and is the precursor to all ROS. The latter at physiological levels are essential for the normal functioning of the body [20]. However, in diabetes, constant influx of glucose in brain, eye, nerves, etc., causes an overproduction of superoxide as described: The excess electron donors produced during the Krebs cycle generate high mitochondrial membrane potential by pumping protons across the inner mitochondrial membrane. The high mitochondrial membrane potential inhibits electron transport at complex III, increases the half-life of the 
free radical intermediate of coenzyme $Q$, which reduces $\mathrm{O}_{2}$ to superoxide. High levels of superoxide lead to overproduction of ROS, which in turn trigger other molecular pathways of ROS production such as polyol pathway [21-23], Advanced Glycation End Products (AGE) formation [24-26], protein kinase C activation [27], and hexosamine pathway [28,29], which in turn propagate more ROS. Though small fluctuations in the steadystate concentration of these oxidants may actually play a role in intracellular signaling [20], uncontrolled increases lead to free radical mediated chain reactions which target proteins [30], lipids [31], polysaccharides [32], and DNA [33,34], and result in oxidative stress. Our published data show that inhibition of mCA with topiramate slows the rate of respiration, ROS production, and oxidative stress in brain pericytes $[11,13]$.

Topiramate protects pericytes from hyperglycemia-induced oxidative stress and apoptosis both in vivo $[10,35]$ and in vitro [11], by inhibiting mCA. There are a total of twelve known active carbonic anhydrases, ten of these are expressed outside of the mitochondria and differ in their tissue distribution, kinetic properties and subcellular localizations [17]. Only two that is CA VA and CA VB, are expressed in the mitochondria [17] and regulate respiration and ROS [13]. Since topiramate inhibits both isoforms of mCA, it is not possible to decipher which of the two plays a predominant role in pericyte apoptosis. Our recent report [36] shows a predominant role of mCA VA in ammonia detoxification whereas both mCA VA and VB contribute equally to gluconeogenesis, giving rise to the possibility of either mCA VA or mCA VB playing a more prominent role in the regulation of respiration. Our preliminary data showed a reduction in oxidative stress in the brains of mice in which only CA VA was silenced genetically (Supplemental Figure 1); therefore, this study was designed to investigate the contribution of mCA VA in regulation of ROS production and apoptosis in brain pericytes.

We now report that mCA VA alone modulates ROS production and pericytes apoptosis. As illustrated in Figures 2 and 4, the overexpression of mCA VA significantly increased ROS (Figure 2) and percent apoptotic cells (Figure 4). Furthermore, inhibition of mCA VA with topiramate reduced ROS (Figure 2) to normal levels and rescued pericytes (Figure 4).

Topiramate is in clinical use for treatment of other diseases [22-37], and can be potentially used to inhibit mCA VA to protect the microvasculature of the brain from hyperglycemia-induced damage. However, topiramate has serious side effects owing to its ability to inhibit other metabolic pathways [22-38] and nonmitochondrial CA [39]. Since mCA VA alone is sufficient to protect pericytes from glucose toxicity, drugs can be designed to target this specific isoform. These mCA VA specific drugs will be less toxic and may be better tolerated by the patients.

These drugs may also prove useful in preventing diabetic damage to other insulin-insensitive tissues such as the eye and the kidney [4], since events similar to the ones caused by hyperglycemia-induced oxidative stress in the brain microvasculature; also occur in the capillary beds of these other tissues [15]. Loss of pericytes in retinal microvasculature precedes endothelial cell death and blindness [40,41]. In the kidney, hyperglycemia-induced renal vascular cells loss [42-44] eventually leads to diabetic kidney disease.

\section{PERICYTE}

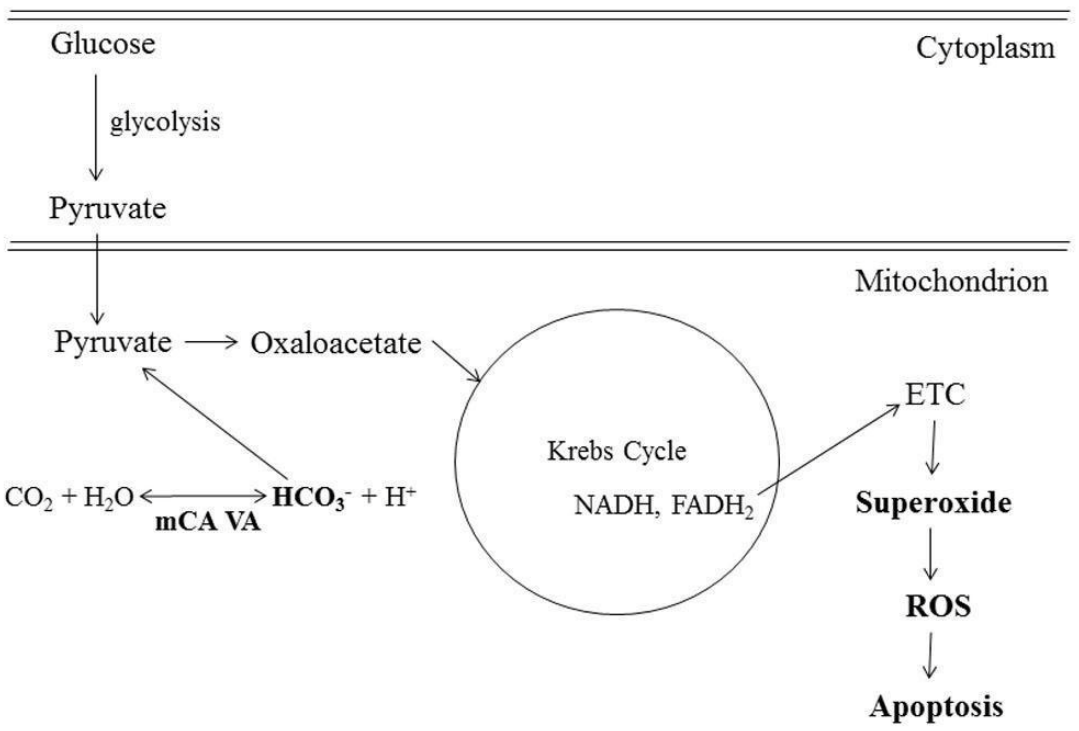

Figure 5: Role of mitochondrial CA VA in ROS production and apoptosis in brain Pericytes. As described in the text mCA VA regulate the rate of respiration by providing $\mathrm{HCO}_{3}$ essential for the conversion of pyruvate to oxaloacetate. Oxaloacetate is essential for Krebs cycle, Electron Transport Chain (ETC), and ATP production. Superoxide is generated as a byproduct of ETC reactions. In diabetes, excess superoxide is produced in brain pericytes due to a constant influx of glucose. Superoxide is precursor to all reactive species (ROS), the latter cause oxidative stress and diabetic damage. Blockage of mCA VA, cuts down the production of $\mathrm{HCO}_{3}^{-}$thus reduces superoxide production, ROS, and pericyte apoptosis. 


\section{Acknowledgement}

This study was supported by the National Institute of Health grant R01DK083485 (G.N.S and W.A.B) and Presidential Research Fund Grant 8817 (G.N.S). The authors thank Tracey Baird (Saint Louis University) for editorial assistance.

\section{References}

1. Huber JD. Diabetes, cognitive function, and the blood-brain barrier. Curr Pharm Des. 2008; 14(16):1594-1600.

2. Janson J, Laedtke T, Parisi JE, O’Brien P, Petersen RC, Butler PC. Increased risk of type 2 diabetes in Alzheimer disease. Diabetes 2004; 53(2):474-481.

3. Kalaria RN. Neurodegenerative disease: Diabetes, microvascular pathology and Alzheimer disease. Nat Rev Neurol. 2009; 5(6):305306. doi: $10.1038 /$ nrneurol.2009.72.

4. Balasubramanyam M, Rema M, Premanand C. Biochemical and molecular mechanisms of diabetic retinopathy. Current Science 2002; 83(12):1506-1514.

5. Bek T, Lund-Andersen $H$, Hansen AB, Johnsen KB, Sandbaek $A$ Lauritzen $\mathrm{T}$. The prevalence of diabetic retinopathy in patients with screen-detected type 2 diabetes in Denmark: the ADDITION study. Acta Ophthalmol. 2009; 87(3):270-274. doi: 10.1111/j.17553768.2008.01207.x.

6. Said G. Diabetic neuropathy--a review. Nat Clin Pract Neurol 2007; 3(6):331-340.

7. Huber JD, VanGilder RL, Houser KA. Streptozotocin-induced diabetes progressively increases blood-brain barrier permeability in specific brain regions in rats. Am J Physiol Heart Circ Physiol. 2006; 291(6):H2660-2668.

8. Armulik A, Genove G, Mae M, Nisancioglu MH, Wallgard E, Niaudet C, et al. Pericytes regulate the blood-brain barrier. Nature. 2010; 468(7323):557-561. doi: 10.1038/nature09522.

9. Sagare AP, Bell RD, Zhao Z, Ma Q, Winkler EA, Ramanathan A, et al. Pericyte loss influences Alzheimer-like neurodegeneration in mice. Nat Commun. 2013; 4:2932. doi: 10.1038/ncomms3932.

10. Price TO, Eranki V, Banks WA, Ercal N, Shah GN. Topiramate treatment protects blood-brain barrier pericytes from hyperglycemia-induced oxidative damage in diabetic mice. Endocrinology. 2012; 153(1):362372. doi: 10.1210/en.2011-1638.

11. Shah GN, Price TO, Banks WA, Morofuji Y, Kovac A, Ercal N, et al. Pharmacological inhibition of mitochondrial carbonic anhydrases protects mouse cerebral pericytes from high glucose-induced oxidative stress and apoptosis. J Pharmacol Exp Ther. 2013; 344(3):637-645. doi: 10.1124/jpet.112.201400.

12. Brownlee M. The pathobiology of diabetic complications: a unifying mechanism. Diabetes 2005; 54(6):1615-1625.

13. Shah GN, Morofuji Y, Banks WA, Price TO. High glucose-induced mitochondrial respiration and reactive oxygen species in mouse cerebral pericytes is reversed by pharmacological inhibition of mitochondrial carbonic anhydrases: Implications for cerebral microvascular disease in diabetes. Biochem Biophys Res Commun. 2013; 440(2):354-358. doi: 10.1016/j.bbrc.2013.09.086.

14. Liu Y, Fiskum G, Schubert D. Generation of reactive oxygen species by the mitochondrial electron transport chain. J Neurochem 2002; 80(5):780-787.
15. Giacco F, Brownlee M. Oxidative stress and diabetic complications. Circ Res. 2010; 107(9):1058-1070. doi: 10.1161/ CIRCRESAHA.110.223545.

16. Ott M, Gogvadze V, Orrenius S, Zhivotovsky B. Mitochondria, oxidative stress and cell death. Apoptosis 2007; 12(5):913-922.

17. Shah GN, Hewett-Emmett D, Grubb JH, Migas MC, Fleming RE, Waheed $A$, et al. Mitochondrial carbonic anhydrase CA VB: differences in tissue distribution and pattern of evolution from those of CA VA suggest distinct physiological roles. Proc Natl Acad Sci U S A 2000; 97(4):1677-1682.

18. Hunter DJ, Gurney EG. The genomic instability associated with integrated simian virus 40 DNA is dependent on the origin of replication and early control region. J Virol 1994; 68(2):787-796.

19. Nagao Y, Srinivasan M, Platero JS, Svendrowski M, Waheed A, Sly WS. Mitochondrial carbonic anhydrase (isozyme $\mathrm{V}$ ) in mouse and rat: cDNA cloning, expression, subcellular localization, processing, and tissue distribution. Proc Natl Acad Sci U S A 1994; 91(22):1033010334.

20. Droge W. Free radicals in the physiological control of cell function. Physiol Rev 2002; 82(1):47-95.

21. Das EN, Ulusu NN, Karasu C, Dogru B. Adenosine triphosphatase activity of streptozotocin-induced diabetic rat brain microsomes. Effect of vitamin E. Gen Physiol Biophys 2004; 23(3):347-355.

22. Leniger $\mathrm{T}$, Thone J, Wiemann M. Topiramate modulates $\mathrm{pH}$ of hippocampal CA3 neurons by combined effects on carbonic anhydrase and Cl-/HCO3- exchange. Br J Pharmacol. 2004; 142(5):831-842.

23. Nishimura C, Hotta Y, Gui T, Seko A, Fujimaki T, Ishikawa T, et al. The level of erythrocyte aldose reductase is associated with the severity of diabetic retinopathy. Diabetes Res Clin Pract 1997; 37(3):173-177.

24. Du XL, Edelstein D, Rossetti L, Fantus IG, Goldberg H, Ziyadeh F, et al. Hyperglycemia-induced mitochondrial superoxide overproduction activates the hexosamine pathway and induces plasminogen activator inhibitor-1 expression by increasing Sp1 glycosylation. Proc Natl Acad Sci U S A 2000; 97(22):12222-12226.

25. Hammes HP, Martin S, Federlin K, Geisen K, Brownlee M. Aminoguanidine treatment inhibits the development of experimental diabetic retinopathy. Proc Natl Acad Sci USA. 1991; 88(24):1155511558.

26. Nakamura S, Makita Z, Ishikawa S, Yasumura K, Fujii W, Yanagisawa $\mathrm{K}$, et al. Progression of nephropathy in spontaneous diabetic rats is prevented by OPB-9195, a novel inhibitor of advanced glycation. Diabetes 1997; 46(5):895-899.

27. Koya D, King GL. Protein kinase $C$ activation and the development of diabetic complications. Diabetes 1998 ;47(6):859-866.

28. Nerlich AG, Sauer U, Kolm-Litty V, Wagner E, Koch M, Schleicher ED. Expression of glutamine:fructose-6-phosphate amidotransferase in human tissues: evidence for high variability and distinct regulation in diabetes. Diabetes 1998; 47(2):170-178.

29. Schleicher ED, Weigert C. Role of the hexosamine biosynthetic pathway in diabetic nephropathy. Kidney Int Suppl 2000; 77:S13-S18.

30. Stadtman ER, Levine RL. Protein oxidation. Ann N Y Acad Sci 2000; 899:191-208.

31. Rubbo H, Radi R, Trujillo M, Telleri R, Kalyanaraman B, Barnes S, et al. Nitric oxide regulation of superoxide and peroxynitrite-dependent 
lipid peroxidation. Formation of novel nitrogen-containing oxidized lipid derivatives. J Biol Chem 1994; 269(42):26066-26075.

32. Kaur H, Halliwell B. Evidence for nitric oxide-mediated oxidative damage in chronic inflammation. Nitrotyrosine in serum and synovial fluid from rheumatoid patients. FEBS Lett 1994; 350(1):9-12.

33. LeDoux SP, Driggers WJ, Hollensworth BS, Wilson GL. Repair of alkylation and oxidative damage in mitochondrial DNA. Mutat Res 1999; 434(3):149-159.

34. Richter C, Park JW, Ames BN. Normal oxidative damage to mitochondrial and nuclear DNA is extensive. Proc Natl Acad Sci U S A 1988; 85(17):6465-6467.

35. Price TO, Farr SA, Niehoff ML, Ercal N, Morley JE, Shah GN. Protective Effect of Topiramate on Hyperglycemia-Induced Cerebral Oxidative Stress, Pericyte Loss and Learning Behavior in Diabetic Mice. International Library of Diabetes \& Metabolism 2015; 1:6-12.

36. Shah GN, Rubbelke TS, Hendin J, Nguyen H, Waheed A, Shoemaker JD, et al. Targeted mutagenesis of mitochondrial carbonic anhydrases VA and VB implicates both enzymes in ammonia detoxification and glucose metabolism. Proc Natl Acad Sci USA. 2013; 110(18):74237428. doi: 10.1073/pnas.1305805110.

37. Puscas I, Buzas G. Treatment of duodenal ulcers with ethoxzolamide, an inhibitor of gastric mucosa carbonic anhydrase. Int J Clin Pharmacol Ther Toxicol. 1986; 24(2):97-99.
38. Deutsch SI, Schwartz BL, Rosse RB, Mastropaolo J, Marvel CL, Drapalski AL. Adjuvant topiramate administration: a pharmacologic strategy for addressing NMDA receptor hypofunction in schizophrenia. Clin Neuropharmacol. 2003; 26(4):199-206.

39. Nishimori I, Vullo D, Innocenti A, Scozzafava A, Mastrolorenzo A, Supuran CT. Carbonic anhydrase inhibitors: inhibition of the transmembrane isozyme XIV with sulfonamides. Bioorg Med Chem Lett 2005; 15(17):3828-3833.

40. Kowluru RA, Abbas SN. Diabetes-induced mitochondrial dysfunction in the retina. Invest Ophthalmol Vis Sci 2003; 44(12):5327-5334.

41. Miller AG, Smith DG, Bhat M, Nagaraj RH. Glyoxalase I is critical for human retinal capillary pericyte survival under hyperglycemic conditions. J Biol Chem. 2006; 281(17):11864-11871.

42. Kang BP, Frencher S, Reddy V, Kessler A, Malhotra A, Meggs LG. High glucose promotes mesangial cell apoptosis by oxidant-dependent mechanism. Am J Physiol Renal Physiol. 2003; 284(3):F455-F466.

43. Nakamura T, Ushiyama C, Suzuki S, Hara M, Shimada N, Ebihara I, et al. Urinary excretion of podocytes in patients with diabetic nephropathy. Nephrol Dial Transplant 2000; 15(9):1379-1383.

44.Steffes MW, Schmidt D, McCrery R, Basgen JM; International Diabetic Nephropathy Study Group. Glomerular cell number in normal subjects and in type 1 diabetic patients. Kidney Int 2001; 59(6):2104-2113.

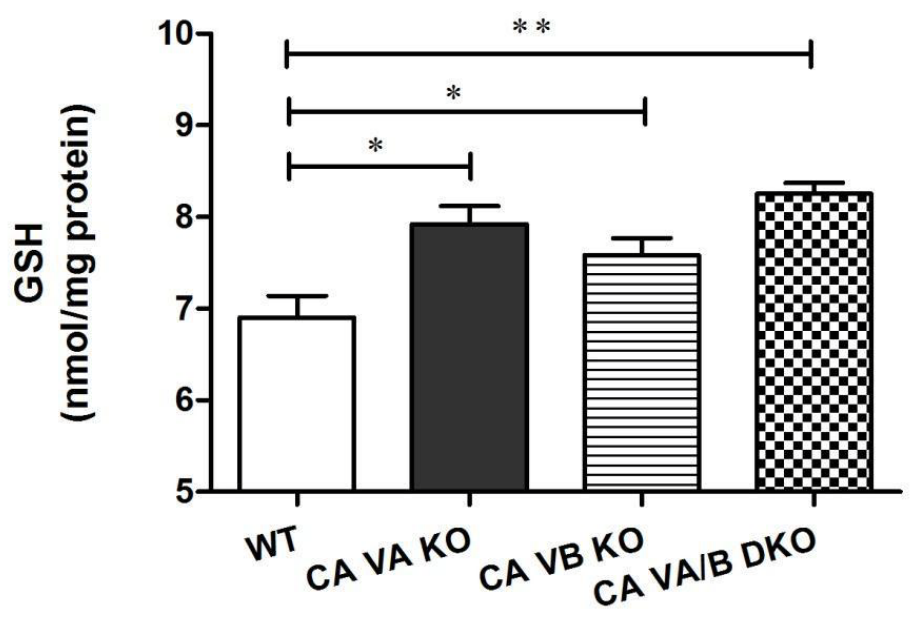

Supplemental Figure 1: Genetic knockout of mCA genes reduces oxidative stress in the mouse brain. The measure of oxidative stress was reduced glutathione (GSH), direct scavenger of ROS. Low levels of GSH indicate oxidative stress. The levels of GSH were significantly higher in CA VA, CA VB, and CA VA/B double knockout (DKO) mice. We selected CA VA for the current study because GSH in CA VA knockout was higher than CA VB though not statistically significant. 